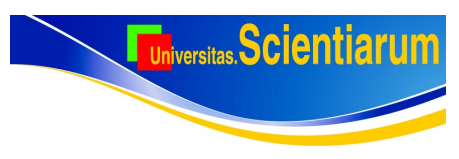

Disponible en línea en:

www.javeriana.edu.co/universitas_scientiarum

2010, Vol. $15 N^{\circ}$ 3: $240-250$

SICI: 2027-1352(201009/12)15:3<240:PPYACFDPELBDDCGEA>2.0.TS;2-V

Artículo original

doi: 10.11144/Javeriana.SC15-3.paaa

\title{
Proteínas pro-apoptóticas y anti-apoptóticas como factores de pronóstico en Linfoma $B$ difuso de célula grande en adultos
}

\author{
Liliana Martín-Reyes ${ }^{1}$, Sandra Quijano-Gómez ${ }^{2}$, María Mercedes Bravo-Hernández ${ }^{1 *}$ \\ ${ }^{1}$ Grupo de Investigación en Cáncer y Agentes Infecciosos. Instituto Nacional de Cancerología. Bogotá, D.C., Colombia. \\ ${ }^{2}$ Grupo de Inmunobiología y Biología Celular. Pontificia Universidad Javeriana. Bogotá, D.C., Colombia. \\ *mbravo@cancer.gov.co
}

Recibido: 06-10-2010; Aceptado: 03-12-2010

\begin{abstract}
Resumen
Objetivo. El propósito de este estudio fue evaluar en pacientes con LBDCG la expresión de las proteínas anti-apoptóticas Bcl-2 y Bcl-X y de las proteínas pro-apoptóticas Bad y Bax y su asociación con la supervivencia. Materiales y métodos. Se analizaron biopsias de 28 pacientes con diagnóstico de LBDCG. La expresión de los reguladores apoptóticos se evaluó mediante western blot. La asociación entre la expresión de las proteínas y la supervivencia fue analizada mediante el método de Kaplan-Meier y la prueba log-rank. Resultados. Las proteínas Bcl-2, Bak, Bad y Bcl-x se encontraron expresadas en el 78,8 \%; 71,4 \%; 64,3\% y 50 \% de los casos de LBDCG respectivamente. No encontramos asociación entré la presencia de las proteínas o sus niveles de expresión y la supervivencia total. La presencia de las proteínas Bad y Bcl-xL se asoció con una mayor supervivencia libre de enfermedad (33,3\% vs. 20,0\%, p LR test $=0,003 ; 42,9 \%$ vs. $14,3 \%$, $\mathrm{p}$ LR test $=0,03$ respectivamente). Niveles altos de expresión de Bad y de Bcl-X se asociaron con una supervivencia libre de enfermedad mayor (35,7\% vs. $21,4 \%$, p LR test $=0,012$ y 42,9\% vs. $14,3 \%$, p LR test $=0,045$ respectivamente). Conclusión. Dado que la expresión de la proteína Bad en los tumores se asoció con una mayor supervivencia libre de enfermedad, los pacientes con bajos niveles de expresión de esta proteína podrían ser beneficiados en un futuro con terapias orientadas a inhibir las moléculas anti apoptóticas Bcl-xL y Bcl-2 mediante el empleo de moléculas que se unen específicamente al dominio BH3.
\end{abstract}

Palabras clave: Linfoma B Difuso de Célula grande, proteína Bcl-2, proteína Bcl-X , proteína Bad, proteína Bax, análisis de supervivencia.

\begin{abstract}
Pro-apoptotic and anti-apoptotic proteins as prognostic factors in diffuse large B-cell lymphoma in adults. Objective. Our purpose was to evaluate the expression of antiapoptotic proteins Bcl-2 and Bcl-x and pro-apoptotic proteins Bad and Bax and their association with survival, in patients with DLBCL. Materials and methods. We ànalyzed biopsies from 28 patients diagnosed with DLBCL. The expression of the apoptotic regulators was assessed by western blot. The association between protein expression and survival was analyzed by the Kaplan-Meier method and the log-rank test. Results. Bcl-2, Bak, Bad and Bcl-x proteins were expressed in 78.8, 71.4, 64.3 and 50\% of the DLBCL cases, respectively. We found no association between the presence of proteins or their expression levels and overall survival. Both Bad and Bcl-x were associated with higher disease-free survival (33.3\% vs. 20.0\%, p LR test $=0,003 ; 42.9 \%$ vs. $14.3 \%$, p LR test $=0.03$, respectively). High expression levels of Bad and Bcl-x were associated with a higher disease-free survival ( $35.7 \%$ vs. $21.4 \%$, p LR test $=0.012$ y $42.9 \%$ vs. $14.3 \%$, p LR test $=0.045$, respectively). Conclusion. Given that expression of the Bad protein in tumors was related to a higher disease-free survival, patients with low expression levels of Bad could be candidates in future therapies oriented towards the inhibition of the anti-apoptotic proteins Bcl-x and Bcl- 2 by using molecules that bind specifically to the $\mathrm{BH} 3$ domain.
\end{abstract}

Key words: diffuse large B-cell lymphoma, Bcl-2 protein, Bcl-X protein, Bad protein, Bax protein, survival analysis 


\begin{abstract}
Resumo
Proteínas pró-apoptóticas e anti-apoptóticas como fatores de prognóstico no Linfoma B difuso de célula grande em adultos. Objetivo. O objetivo deste estudo foi avaliar em pacientes com LBDCG a expressão das proteínas anti-apoptótica Bcl-2 e Bcl-X e das proteínas pró-apoptóticas Bad e Bax e sua associação com a sobrevivência. Materiais e métodos. Foram analisadas biópsias de 28 pacientes com diagnóstico de LBDCG. A expressão de reguladores de apoptose foi avaliada por Western blot. A associação entre a expressão das proteínas e a sobrevivência foi analisada pelo método de Kaplan-Meier e o teste log-rank. Resultados. As proteína Bcl-2, Bak, Bad e Bcl-x foram expressas em 78,8\%, 71,4\%, 64,3\% e 50\% dos casos de LBDCG, respectivamente. Nós não encontramos associação entre a presença das proteínas ou de seus níveis de expressão e a sobrevivência total. A presença das proteínas Bad e Bcl-x foi associada à maior sobrevivência livre da doença (33,3\% vs. 20,0\%, p LR teste $=0,003 ; 42,9 \%$ vs. 14,3\%, p LR teste $=0,03$, respectivamente). Altos níveis de expressão de Bad e de Bcl-X foram associados com uma sobrevivência livre da doença maior (35,7\% vs. 21,4\%, p LR teste $=0,012$ e $42,9 \%$ vs. $14,3 \%$, p LR teste $=0,045$, respectivamente). Conclusão. Uma vez que a expressão da proteína Bad em tumores foi associada com uma maior sobrevivência livre de doença, os pacientes com baixos níveis de expressão dessa proteína poderiam ser beneficiados num futuro com terapias orientadas a inibir as moléculas anti-apoptóticas Bcl-x e Bcl-2 utilizando moléculas que se ligam especificamente ao domínio BH3.
\end{abstract}

Palavras-chave: Linfoma B Difuso de Célula grande (LBDCG), proteína Bcl-2, proteína Bcl-X, proteína Bad, proteína Bax, análise de sobrevivência.

\section{Introducción}

El linfoma B difuso de célula grande (LBDCG) es el subtipo de linfomas no Hodgkin (LNH) más común en los países occidentales, representa entre el 30 y el $40 \%$ de todos los LNH diagnosticados en adultos, en la población pediátrica representa entre el 15 y el $20 \%$ (1). Según los criterios diagnósticos incluidos en la clasificación de neoplasias hematológicas de la OMS, los LBDCG se subdividen en distintas entidades desde el punto de vista morfológico, inmunofenotípico y genético con diferencias importantes en comportamiento clínico, respuesta al tratamiento y supervivencia (2).

El pronóstico del LBDCG varía según sus características clínicas definidas por el índice de pronóstico internacional (IPI) que está basado en cinco factores pronóstico independientes que incluyen: edad al momento del diagnóstico, estadio clínico, infiltración de sitios extraganglionares, nivel sérico de lactato deshidrogenasa, y estado general (3). Existe una variabilidad considerable en la supervivencia de pacientes con idéntico IPI, ésta se atribuye a la heterogeneidad genética y molecular de esta neoplasia (4). La evaluación de los perfiles de expresión génica ha contribuido al entendimiento de la heterogeneidad de los LBDCG mediante la identificación de al menos tres subgrupos principales con un origen celular diferente: el subgrupo denominado similar a centro germinal B (CGB) que presenta alta expresión de genes característicos de los linfocitos B del centro germinal, un segundo subgrupo similar a linfocitos B activados con características de expresión similares a las observadas en linfocitos $\mathrm{B}$ activados (ABC), y un tercer subgrupo denominado tipo 3 que muestra heterogeneidad en el patrón de expresión génica. Estos subgrupos muestran diferencias en respuesta al trata- miento con un pronóstico más favorable para el subtipo similar a LB de centro germinal (5). Los microarreglos de $\mathrm{ADN}$ aun no se emplean de manera rutinaria en la clínica debido a su costo y a que están disponibles en pocos centros y además requieren la evaluación de tejido fresco o congelado lo que dificulta su aplicación. A partir de los hallazgos en expresión diferencial de mRNA de varios marcadores se ha comprobado que los LDDCG se pueden clasificar en dos grupos de pronóstico: los de centro germinal y los que no corresponden a centro germinal con base en la detección por immunohistoquímica de CD10, bcl-6 y MUM1 (6). Sin embargo, los subtipos GBC y ABC no fueron incorporados formalmente en la clasificación de la OMS del 2008 debido a la no disponibilidad del perfil de expresión génica como test diagnóstico de rutina y a que la correlación entre la inmunohistoquímica y los estudios de expresión génica es imperfecta. Adicionalmente estos subtipos aun no direccionan la terapia (1).

La apoptosis y la regulación del ciclo celular juegan un papel importante en la patogénesis y progresión tumoral en pacientes con LBDCG, los tumores que son intrínsecamente resistentes a la quimioterapia presentan alteraciones genéticas que afectan genes reguladores de la apoptosis lo que los hace incapaces de activar la maquinaria apoptótica (7). La proteína Bcl-2 hace parte de una familia de proteínas relacionadas que contienen regiones de homología denominadas dominios de homología con Bcl2 (BH), algunas de ellas son anti-apoptóticas y contienen cuatro dominios de homología $\mathrm{BH}_{1-4}\left(\mathrm{Bcl}-2, \mathrm{Bcl}-\mathrm{X}_{\mathrm{L}}\right)$ y otras son pro-apoptóticas y contienen tres dominios $\mathrm{BH}_{1-3}$ (Bax, Bak, Bcl- $\mathrm{X}_{\mathrm{S}} \mathrm{y}$ otras), existe un tercer grupo proapoptótico con un único dominio de homología $\mathrm{BH}_{3}(\mathrm{Bad}$, Bid, Bik y otras) (8). La expresión de las proteínas de la familia Bcl-2 es variable y heterogénea en los LBDCG (9- 
11). La proteína Bcl-2 es una de las más estudiadas en LBDCG, su expresión ha sido reportada en 24 a $60 \%$ de estos (4), y se asocia con menor supervivencia y quimioresistencia $(12,13)$, sin embargo en varios estudios no se ha encontrado esta asociación $(14,15)$. Aunque la expresión de otras proteínas de la familia Bcl-2 como Bad, Bax y Bcl- $\mathrm{X}_{\mathrm{L}}$ ha sido menos estudiada en LBDCG, también se ha encontrado asociada a resistencia a la quimioterapia y menor supervivencia $(9,10,16)$. En el presente estudio se evaluó la expresión de las proteínas antiapoptóticas $\mathrm{Bcl}-2$ y $\mathrm{Bcl}-\mathrm{X}_{\mathrm{L}}$ y de las proteínas proapoptóticas Bad y Bax en biopsias de pacientes con LBDCG y su asociación con la supervivencia total y la supervivencia libre de enfermedad.

\section{Materiales y métodos}

\section{Especímenes clínicos}

Se incluyeron biopsias provenientes de 34 pacientes adultos con diagnóstico de LBDCG de localización ganglionar tratados en el Instituto Nacional de Cancerología de Bogotá, Colombia (INC) entre 1995 y 1999. La biopsias fueron recolectadas al momento del ingreso y conservadas a $-80^{\circ} \mathrm{C}$ desde el momento de su toma. El diagnóstico de los casos fue realizado por el hematopatólogo del Instituto Nacional de Cancerología con los parámetros vigentes para la fecha de recolección de las biopsias. Los datos clínicopatológicos, modalidades de tratamiento y datos de seguimiento se tomaron de las historias clínicas. Este artículo es producto de un proyecto de investigación que fue presentado al Comité de Ética e Investigaciones del Instituto Nacional de Cancerología y fue aprobado para su realización y financiado con recursos de inversión de la nación a través del Departamento Nacional de Planeación. Los pacientes consintieron de manera voluntaria su participación en el estudio.

\section{Obtención de extractos proteicos}

Las biopsias fueron descongeladas y disgregadas con bisturí en solución de lisis que contenía Igepal CA-630 al $1 \%$, EDTA $(2 \mathrm{mM})$, pepstatina A $(1 \mu \mathrm{g} / \mathrm{mL})$, aprotinina (2 $\mu \mathrm{g} / \mathrm{mL})$ y PMSF $(100 \mu \mathrm{g} / \mathrm{mL})$ en tampón fosfato salino (PBS). La suspensión fue sometida a siete ciclos de ultrasonido, con pulsos de $1 \mathrm{~min}$. y recesos de $30 \mathrm{~s}$., posteriormente se centrifugó, se recuperó el sobrenadante y se almacenó a $-20^{\circ} \mathrm{C}$ hasta su uso. Para emplear como control positivo para el western blot, se preparó un extracto a partir de la línea celular AGS (ATCC CRL-1739) derivada de adenocarcinoma gástrico.

\section{Western blot}

Los extractos proteicos fueron separados mediante SDSPAGE al 12,5\%. En cada gel se incluyó un patrón de peso molecular (BIO-RAD, Low Range). Las proteínas se transfirieron del gel a una membrana de Inmobilon-P Millipore ${ }^{\circledR}$ empleando un sistema de transferencia semiseco. Se bloquearon los sitios libres de la membrana mediante incubación con leche descremada al $2 \%$ y Tween 20 al 0,05\% en PBS (PBS-tween-leche) durante dos horas. Las membranas fueron lavadas 3 veces con PBS-tween al $0.1 \%$ por 5 y posteriormente incubadas por 2 horas a temperatura ambiente con un panel de 7 anticuerpos marca Santa Cruz Biotechnologies específicos para Bad, (Bak sc-832), (sc7869), Bcl-x (sc-509), Bag-1 (sc-939), Bax (sc-7480) Bcl2 (sc-509) y Actina (sc-7210). Posteriormente, la membrana se lavó cuatro veces, se incubó por 1 hora con el anticuerpo secundario conjugado con peroxidasa en una dilución 1:500 y finalmente, luego de 3 lavados similares a los anteriores, las bandas fueron reveladas con una solución de peróxido de hidrógeno y aminoetilcarbazol. Como control positivo se evaluó Bcl-2 en extractos proteicos de la línea celular AGS, y como control interno de cada muestra se determinó la Actina. Las membranas fueron analizadas en un densitómetro (Bio Rad, modelo GS-670). Cada banda fue caracterizada por su peso molecular usando una curva de calibración. Adicionalmente se determinó la densidad óptica de cada banda (volumen $/ \mathrm{mm}^{2}$ ) que fue corregida con el valor determinado para la banda de Actina en cada caso.

\section{Análisis estadístico}

Los resultados fueron analizados con el programa SPSS. La asociación entre la expresión de las proteínas y la supervivencia fue analizada mediante el método de KaplanMeier y la prueba log-rank. Se consideró una diferencia significativa cuando la p fue menor a 0,05 . La supervivencia total fue definida como el periodo de tiempo transcurrido entre la fecha de diagnóstico y la fecha de muerte de los pacientes. La supervivencia libre de enfermedad fue calculada como el tiempo transcurrido desde el inicio del tratamiento hasta la reaparición de la enfermedad.

\section{Resultados}

\section{Población}

Se incluyeron en total 34 casos de pacientes con diagnóstico de LBDCG. Sus características clínicobiológicas se presentan en la tabla 1. 
Tabla 1. Características clínicas de los pacientes diagnosticados con LBDCG.

\begin{tabular}{|c|c|c|}
\hline Característica & Frecuencia & Porcentaje (\%) \\
\hline \multicolumn{3}{|l|}{ Sexo } \\
\hline Masculino & 15 & 44,1 \\
\hline Femenino & 19 & 55,9 \\
\hline \multicolumn{3}{|l|}{ Edad $^{*}$} \\
\hline$<60$ años & 27 & 79,4 \\
\hline$\geq 60$ años & 7 & 20,6 \\
\hline \multicolumn{3}{|l|}{ Estadio clínico } \\
\hline I - II & 17 & 50,0 \\
\hline III-IV & 7 & 20,6 \\
\hline ND & 10 & 29,4 \\
\hline \multicolumn{3}{|l|}{ Hemoglobina } \\
\hline$\geq 12$ ó $13.5 \mathrm{~g} / \mathrm{dL}$ & 11 & 32,3 \\
\hline$<12$ ó $13.5 \mathrm{~g} / \mathrm{dL}^{\mathrm{I}}$ & 16 & 47,1 \\
\hline ND & 7 & 20,6 \\
\hline \multicolumn{3}{|l|}{ Lactato Deshidrogenasa } \\
\hline$\geq 300 \mathrm{UI} / \mathrm{L}$ & 19 & 55,9 \\
\hline$<300 \mathrm{UI} / \mathrm{L}$ & 13 & 38,2 \\
\hline ND & 2 & 5,9 \\
\hline \multicolumn{3}{|c|}{ Infiltración fuero de ganglios } \\
\hline $\mathrm{Si}$ & 12 & 35,3 \\
\hline No & 22 & 64,7 \\
\hline \multicolumn{3}{|l|}{ Tratamiento } \\
\hline Quimioterapia & 18 & 52,9 \\
\hline Radioterapia & 2 & 5,9 \\
\hline Ambas & 14 & 41,2 \\
\hline
\end{tabular}

ND: No determinado, ${ }^{*}$ Rango de edad de la población analizada 30-84 año,. ${ }^{¥}$ Valor de referencia hemoglobina: $=12 \mathrm{~g} / \mathrm{dL}$ para las mujeres $\mathrm{y}=13,5 \mathrm{~g} / \mathrm{dL}$ para los hombres, * Estos valores con respecto al total de la población: 34 pacientes

\section{Expresión de proteínas pro- y anti-apoptóticas}

La expresión de proteínas fue analizada en 28/34 muestras, debido a que en 6 muestras no se obtuvo un extracto proteico en la concentración requerida para los ensayos En la figura 1 se presentan los resultados representativos de western blot para dos casos. En la Tabla 2 se presentan los resultados obtenidos para la totalidad de los casos analizados.

Tabla 2. Porcentaje de expresión de proteínas pro- y anti-apoptóticas en los casos evaluados de LBDCG.

\begin{tabular}{lll}
\hline Proteina & $\mathbf{n}^{\mathbf{a}}$ & $\mathbf{\%}$ \\
\hline Bcl-2 & 22 & 78,6 \\
Bak & 20 & 71,4 \\
Bad & 18 & 64,3 \\
Bcl-xL & 14 & 50,0 \\
\hline
\end{tabular}

a Para una población de 28 muestras

La proteína Bcl-2 fue la que se observó con más frecuencia en las muestras, seguida de las proteínas Bak y Bad. La proteína Bax sólo fue observada en una de las muestras, mientras que la proteína Bag no fue observada en ninguna, por lo que estas dos proteínas fueron excluidas de los análisis posteriores.

En la figura 2 se presenta la distribución de los niveles de expresión en los 28 casos de LBDCG para cada una de las
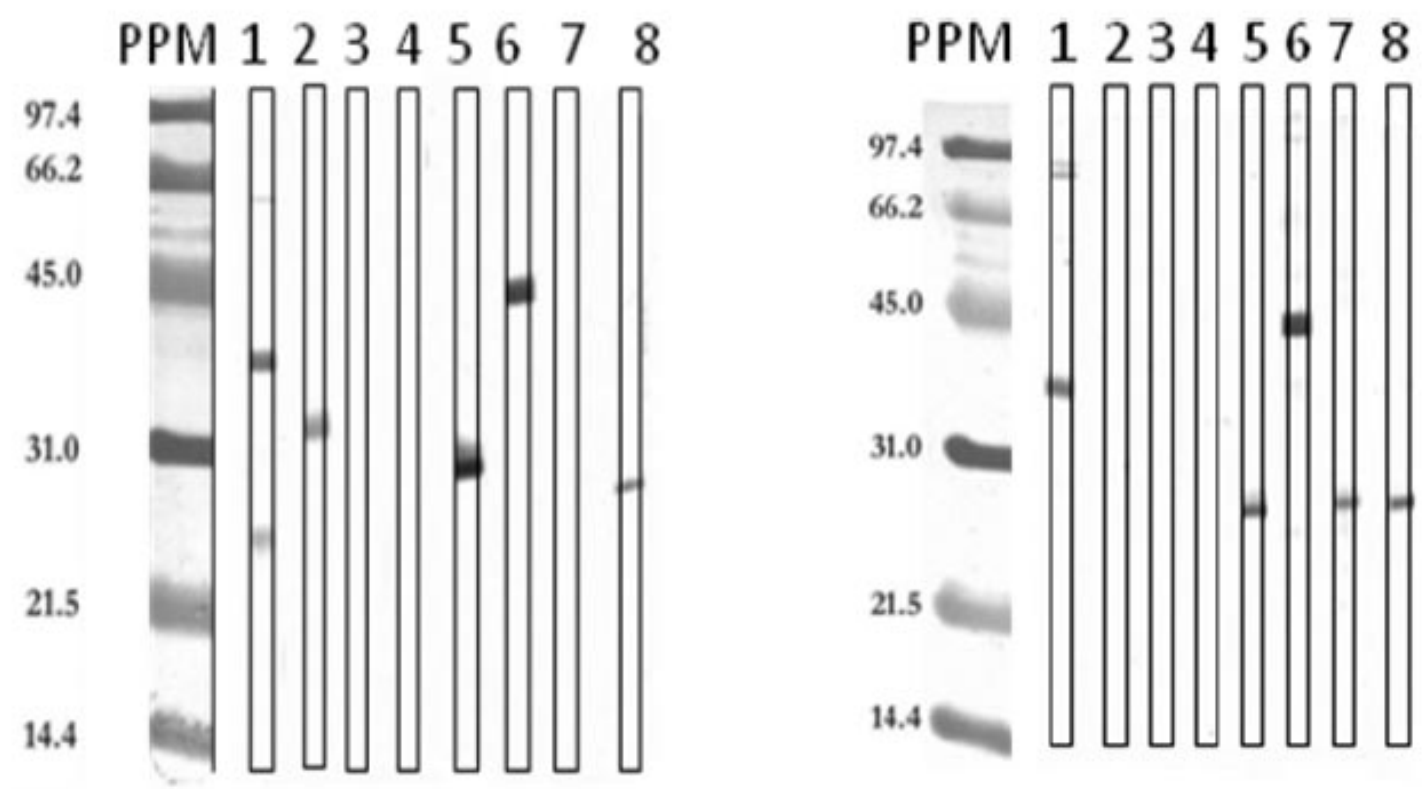

Figura 1. Expresión de proteínas pro-apoptóticas y anti-apoptóticas en dos casos representativos de LBDCG: 1. Bad, 2. Bclx, 3. Bag, 4. Bax, 5. Bcl-2, 6. Actina, 7. Bak, 8. Control positivo: Bcl-2 en células AGS. 


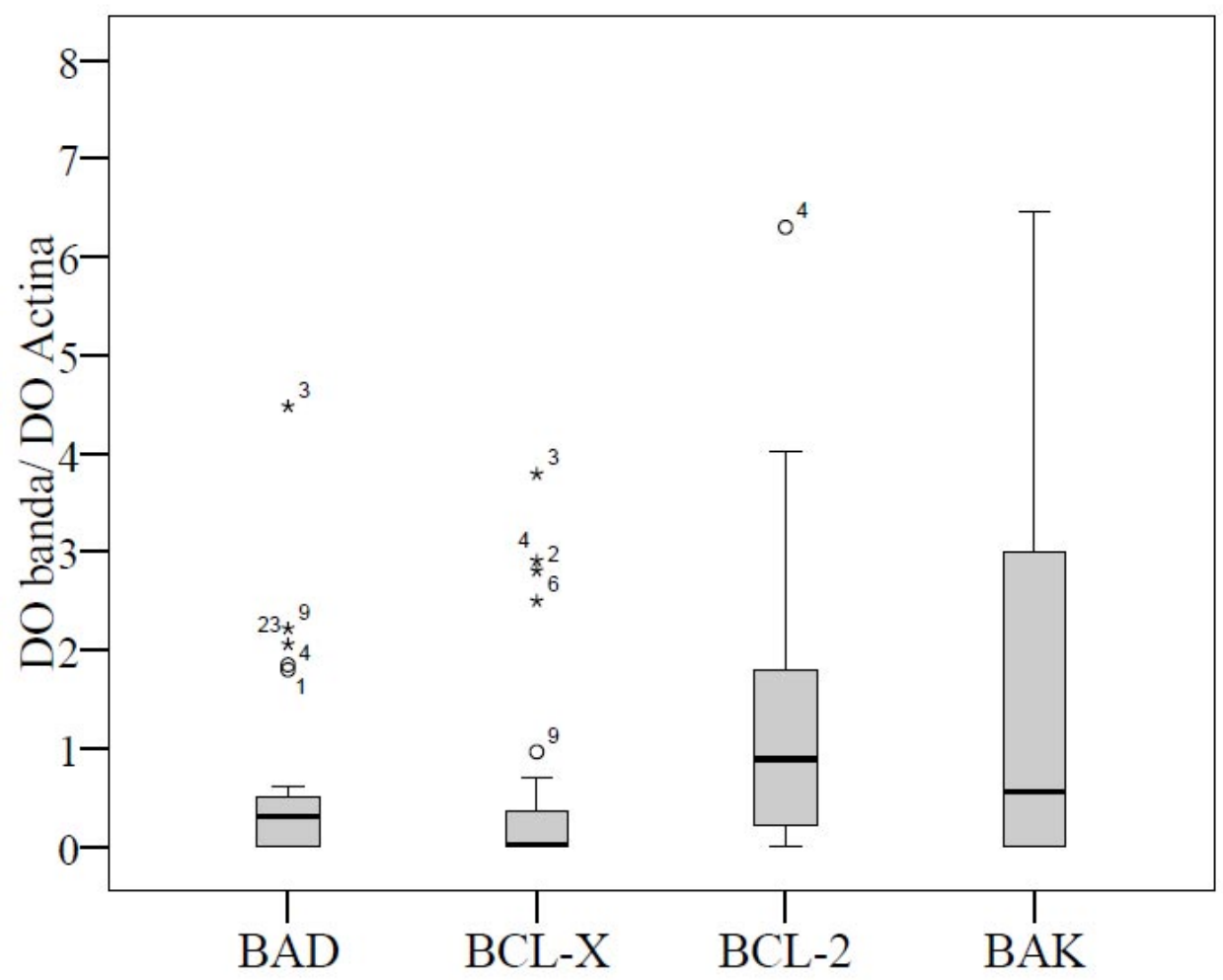

Figura 2. Niveles de expresión de las proteínas pro- y anti-apoptóticas evaluadas en LBDCG.

proteínas analizadas corregidas en relación con la actina. Con el fin de categorizar los resultados de expresión de cada proteína, se tomaron los valores por encima del percentil 50 como de alta expresión y aquellos por debajo o iguales a este percentil como de baja expresión.

\section{Supervivencia total y supervivencia libre de enfermedad}

El tiempo medio de supervivencia total de los pacientes evaluados fue de 26,3 meses y el de supervivencia libre de enfermedad fue de 16,9 meses. De los 34 pacientes evaluados 24 presentaron recaída de la enfermedad $(70,6 \%)$ durante el seguimiento y 19 murieron $(55,9 \%)$.

Se realizó un análisis univariado de las variables clínicas de los pacientes al momento del diagnóstico en relación con la supervivencia, sin encontrarse asociaciones significativas. En la tabla 3 se presentan los resultados para la supervivencia libre de enfermedad y supervivencia global.

El análisis univariado para la expresión o no expresión de las proteínas Bad, Bcl- $\mathrm{x}_{\mathrm{L}}, \mathrm{Bcl}-2$ y Bak en relación con la supervivencia total y la supervivencia libre de enfermedad se presenta en la tabla 4. La presencia de las proteínas Bad y Bcl$\mathrm{X}_{\mathrm{L}}$ se asoció con una mayor supervivencia libre de enfermedad ( $33,3 \%$ vs. $20,0 \%$, p LR test $=0,003 ; 42,9 \%$ vs. $14,3 \%$, p LR test $=0,03$ respectivamente) (Figura 3, paneles A y B).

Adicionalmente se realizó un análisis de supervivencia en el que la expresión de las proteínas individuales se categorizó de acuerdo al percentil 50 en niveles altos y bajos de expresión (figura 4 paneles A, B y C). El análisis univariado de los niveles de expresión de las proteínas Bad, Bcl-xL, Bcl-2, y Bak en relación con la supervivencia global y la supervivencia libre de enfermedad se presenta en la tabla 5. En concordancia con el análisis según presencia o ausencia de la proteína, se observó que niveles altos de expresión de Bad se asocian con una supervivencia libre de enfermedad mayor comparada con la observada para bajos niveles de expresión de esta proteína (35,7\% vs. $21,4 \%$, p LR test $=0,012$ ) (Tabla 5, Figura 4). La supervivencia libre de enfermedad en los pacientes con altos niveles de Bcl-xL fue de $42.9 \%$ mientras que en pacientes con niveles bajos fue del 14,3\% (p LR test $=0,022$ ). Niveles altos de expresión de la proteína Bcl2 se asociaron significativamente con una mayor supervivencia libre de enfermedad (42,9\% vs. $14,3 \%$, p LR test $=0,045)$ (Tabla 5, Figura 4). 

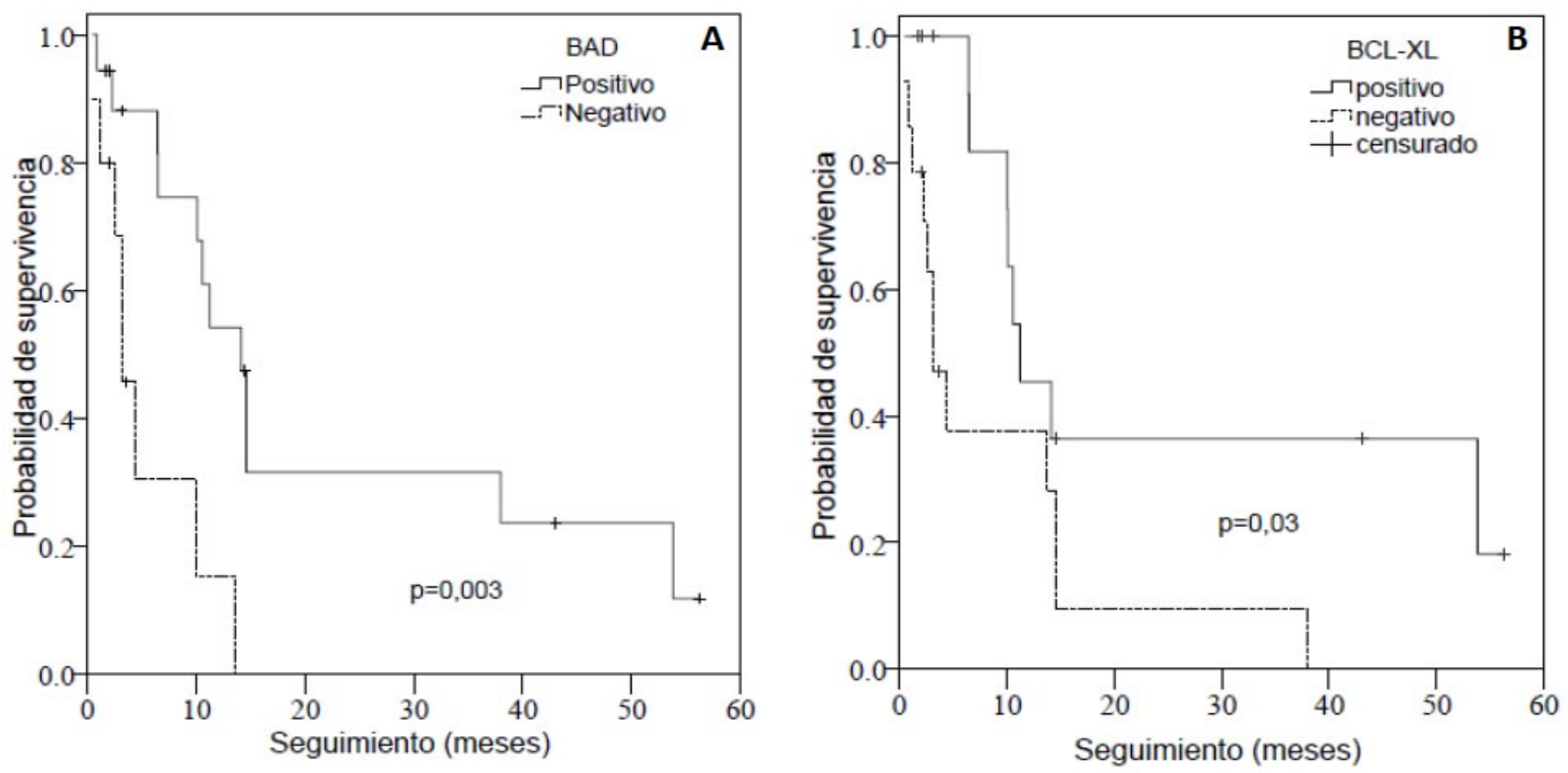

Figura 3. Supervivencia libre de enfermedad a 60 meses de los pacientes con LBDCG evaluada para expresión o no expresión de las proteínas Bad (panel A) y Bcl-XL (panel B).
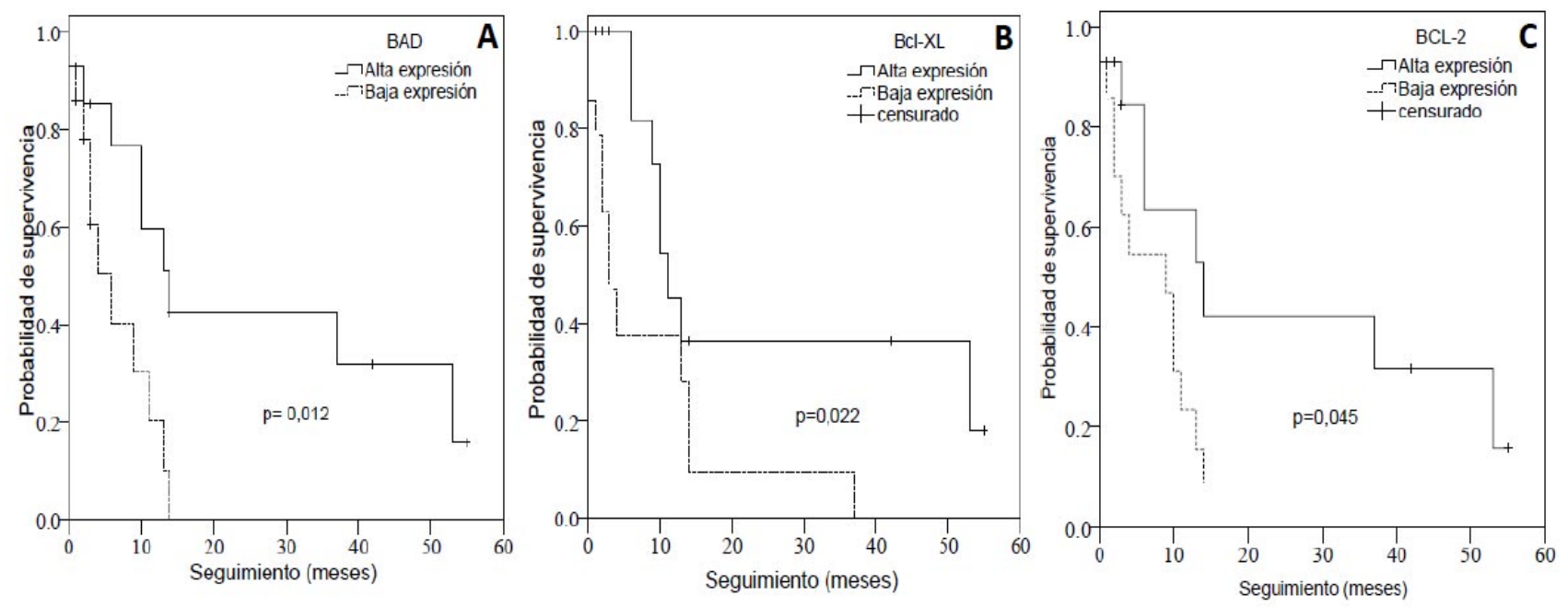

Figura 4. Supervivencia libre de enfermedad a 60 meses de los pacientes con LBDCG evaluada según los niveles de expresión de las proteínas Bad (panel A), $\mathrm{Bcl}_{\mathrm{L}}$ (panel B) y Bcl-2 (panel C). 
Tabla 3. Supervivencia libre de enfermedad y supervivencia global total a 60 meses según las variables clínicas.

\begin{tabular}{|c|c|c|c|c|c|c|c|}
\hline \multirow[t]{2}{*}{$\begin{array}{l}\text { Características } \\
\text { clínicas }\end{array}$} & \multirow[t]{2}{*}{$\mathbf{n}$} & \multicolumn{3}{|c|}{$\begin{array}{c}\text { Supervivencia } \\
\text { libre de enfermedad }\end{array}$} & \multicolumn{3}{|c|}{$\begin{array}{c}\text { Supervivencia } \\
\text { global }\end{array}$} \\
\hline & & $\mathrm{n}$ & $(\%)$ & $\mathrm{p}^{\mathrm{c}}$ & $\mathrm{n}$ & $\%$ & $\mathrm{p}^{\mathrm{c}}$ \\
\hline \multicolumn{8}{|l|}{$\overline{\operatorname{Sexo}}$} \\
\hline Femenino & 12 & 2 & $(16,7)$ & 0,876 & 6 & $(50,0)$ & 0,919 \\
\hline Femenino & 16 & 6 & $(37,5)$ & & 7 & $(43,8)$ & \\
\hline \multicolumn{8}{|l|}{ Edad } \\
\hline$\geq 60$ años & 4 & 0 & $(0,0)$ & 0,984 & 2 & $(50,0)$ & 0,552 \\
\hline$<60$ años & 24 & 8 & $(33,3)$ & & 11 & $(45,8)$ & \\
\hline \multicolumn{8}{|l|}{ Estadio } \\
\hline I-II & 15 & 5 & $(33,3)$ & 0,137 & 6 & $(40,0)$ & 0,831 \\
\hline III-IV & 6 & 0 & $(0,0)$ & & 2 & $(33,3)$ & \\
\hline \multicolumn{8}{|l|}{ LDH } \\
\hline$\geq 300 \mathrm{UI} / \mathrm{L}$ & 16 & 6 & $(37,5)$ & 0,089 & 8 & $(50,0)$ & 0,245 \\
\hline$<300 \mathrm{UI} / \mathrm{L}$ & 10 & 1 & $(10,0)$ & & 3 & $(30,0)$ & \\
\hline \multicolumn{8}{|l|}{ Hemoglobina ${ }^{a}$} \\
\hline$\geq 12 / 13,5 \mathrm{~g} / \mathrm{dL}$ & 10 & 4 & $(40,0)$ & 0,579 & 3 & $(30,0)$ & 0,079 \\
\hline$<12 / 13.5 \mathrm{~g} / \mathrm{dL}$ & 12 & 2 & $(16,7)$ & & 6 & $(50,0)$ & \\
\hline \multicolumn{8}{|l|}{$\mathbf{C E G}^{\mathrm{b}}$} \\
\hline $\mathrm{Si}$ & 11 & 4 & $(36,4)$ & 0,394 & 6 & $(54,5)$ & 0,532 \\
\hline No & 17 & 4 & $(23,5)$ & & 7 & $(41,2)$ & \\
\hline \multicolumn{8}{|l|}{ Tratamiento } \\
\hline Quimioterapia $^{\mathrm{d}}$ & 16 & 6 & $(37,5)$ & 0,746 & 7 & $(43,8)$ & 0,699 \\
\hline Radioterapia & 2 & 0 & $(0,0)$ & & 1 & $(50,0)$ & \\
\hline Ambas & 10 & 2 & $(20,0)$ & & 5 & $(50,0)$ & \\
\hline
\end{tabular}

${ }^{a}$ Valor de referencia: $12 \mathrm{~g} / \mathrm{dL}$ para mujeres y $13,5 \mathrm{~g} / \mathrm{dL}$ para hombres. ${ }^{\mathrm{b}}$ Compromiso extraganglionar. ${ }^{\mathrm{c}} \mathrm{p}$ log-rank test ${ }^{\mathrm{d}}$ Esquema $\mathrm{CHOP}$

Tabla 4. Supervivencia libre de enfermedad y supervivencia global a 60 meses de pacientes con LBDCG de acuerdo a la presencia y ausencia de la banda.

\begin{tabular}{|c|c|c|c|c|c|c|c|}
\hline \multirow[t]{2}{*}{$\begin{array}{l}\text { Características } \\
\text { clínicas }\end{array}$} & \multirow[t]{2}{*}{$\mathrm{n}$} & \multicolumn{3}{|c|}{$\begin{array}{c}\text { Supervivencia } \\
\text { libre de enfermedad }\end{array}$} & \multicolumn{3}{|c|}{$\begin{array}{c}\text { Supervivencia } \\
\text { global }\end{array}$} \\
\hline & & $\mathrm{n}$ & $(\%)$ & $\mathrm{P}^{*}$ & $\mathrm{n}$ & $\%$ & $\mathrm{P}^{*}$ \\
\hline \multicolumn{8}{|l|}{$\overline{\text { Bad }}$} \\
\hline Positivo & 18 & 6 & $(33,3)$ & $\mathbf{0 , 0 0 3}$ & 10 & $(55,6)$ & 0,255 \\
\hline Negativo & 10 & 2 & $(20,0)$ & & 3 & $(30,0)$ & \\
\hline \multicolumn{8}{|l|}{ Bcl-X } \\
\hline Positivo & 14 & 6 & $(42,9)$ & $\mathbf{0 , 0 3}$ & 9 & $(64,3)$ & 0,160 \\
\hline negativo & 14 & 2 & $(14,3)$ & & 4 & $(28,6)$ & \\
\hline \multicolumn{8}{|l|}{ Bcl-2 } \\
\hline Positivo & 22 & 4 & $(31,8)$ & 0,226 & 12 & $(54,5)$ & 0,095 \\
\hline Negativo & 6 & 4 & $(16,7)$ & & 1 & $(16,7)$ & \\
\hline \multicolumn{8}{|l|}{ Bak } \\
\hline Positivo & 20 & 7 & $(30,0)$ & 0,878 & 9 & $(45,0)$ & 0,747 \\
\hline $\begin{array}{l}\text { negativo } \\
\text { p log-rank }\end{array}$ & 8 & 1 & $(25,0)$ & & 4 & $(50,0)$ & \\
\hline
\end{tabular}


Tabla 5. Supervivencia libre de enfermedad y supervivencia global a 60 meses de pacientes con LBDCG de acuerdo a los niveles de expresión de las proteínas pro- y anti-apoptóticas.

\begin{tabular}{|c|c|c|c|c|c|c|c|}
\hline \multirow[t]{2}{*}{ Proteína } & \multirow[t]{2}{*}{$\mathbf{n}$} & \multicolumn{3}{|c|}{$\begin{array}{c}\text { Supervivencia libre de } \\
\text { enfermedad }\end{array}$} & \multicolumn{3}{|c|}{ Supervivencia global } \\
\hline & & $\mathrm{n}$ & $(\%)$ & $\mathrm{P}^{*}$ & $\mathrm{n}$ & $\%$ & $\mathrm{P}^{*}$ \\
\hline \multicolumn{8}{|l|}{ Bad } \\
\hline Alto & 14 & 5 & $(35,7)$ & 0,012 & 10 & $(71,4)$ & 0,062 \\
\hline Bajo & 14 & 3 & $(21,4)$ & & 3 & $(21,4)$ & \\
\hline \multicolumn{8}{|l|}{ Bcl- $X_{L}$} \\
\hline Alto & 14 & 6 & $(42,9)$ & 0,022 & 9 & $(64,3)$ & 0,160 \\
\hline Bajo & 14 & 2 & $(14,3)$ & & 4 & $(28,6)$ & \\
\hline \multicolumn{8}{|l|}{ Bcl-2 } \\
\hline Alto & 14 & 6 & $(42,9)$ & 0,045 & 8 & $(57,1)$ & 0,540 \\
\hline Bajo & 14 & 2 & $(14,3)$ & & 5 & $(35,7)$ & \\
\hline \multicolumn{8}{|l|}{ Bak } \\
\hline Alto & 14 & 5 & $(35,7)$ & 0,464 & 6 & $(42,9)$ & 0,511 \\
\hline 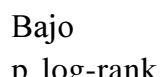 & 14 & 3 & $(21,4)$ & & 7 & $(50,0)$ & \\
\hline
\end{tabular}

\section{Discusión}

El LBDCG es una neoplasia de comportamiento heterogéneo y respuesta variable al tratamiento convencional. En este estudio analizamos mediante western blot la expresión de las proteínas Bad, Bcl- $\mathrm{x}_{\mathrm{L}}$, Bcl-2 y Bax en biopsias pre tratamiento de 28 pacientes con LBDCG y evaluamos su asociación con el pronóstico.

La expresión de la proteína anti-apoptótica Bcl-2 fue observada en el 78,6\% de los casos de LBDCG, en estudios realizados con inmunohistoquímica se reporta expresión de Bcl-2 en un 24 a $60 \%$ de los casos (4). Estas diferencias obedecen a la variabilidad del punto de corte empleado en los estudios de inmunohistoquímica para definir un caso como positivo (10 a 90\%), lo que hace difícil contrastarlos con nuestros resultados en los que la presencia de la banda define la expresión de la proteína.

La proteína $\mathrm{Bcl}-\mathrm{X}_{\mathrm{L}}$ se encontró expresada en un 50,0\% de los casos, la proteína Bak en el 71,4\% y la proteína Bad en el $64,3 \%$. Bai y cols. (11) mediante inmunohistoquímica con un punto de corte del $10 \%$ reportan la expresión de Bcl- $x_{L}$ Bak y Bad en $60 \%, 52 \%$ y $76 \%$ de los casos de LBDCG respectivamente. Saez y cols, (9) empleando microarreglos de tejido y el mismo punto de corte reportan expresión de $\mathrm{Bcl}-\mathrm{x}_{\mathrm{L}}$ en $38,8 \%$ de los casos.
No encontramos asociación entre la presencia de las proteínas analizadas y la supervivencia total. Sin embargo los análisis de la supervivencia libre de enfermedad mostraron que altos niveles de expresión de la proteína pro-apoptótica Bad y de las proteínas anti-apoptóticas Bcl$\mathrm{x}_{\mathrm{L}} \mathrm{y}$ Bcl-2 se asocian con una supervivencia libre de enfermedad mayor en los pacientes evaluados $(\mathrm{p}=0,012$; $\mathrm{p}=0,022$ y $\mathrm{p}=0,045$, respectivamente). El criterio de categorización ausencia y presencia mostró resultados similares. Se encontró asociación de la presencia de la proteína $\mathrm{Bad}(\mathrm{p}=0,003)$ y Bcl-xL $(\mathrm{p}=0,022)$ con una mayor supervivencia libre de enfermedad.

El valor pronóstico de la expresión de Bcl-2 es controversial (17), al igual que en este estudio en varios estudios, en los que se emplea inmunohistoquímica para la evaluación de Bcl-2, no se ha encontrado asociación entre la expresión de Bcl-2 y la supervivencia total (1214). En contraste, existen varios reportes en los que se encuentra esta asociación $(18,19)$. Estas diferencias son explicadas en parte por la variabilidad en las metodologías empleadas (20).

La asociación encontrada entre altos niveles de expresión de Bcl-2 y una mejor supervivencia libre de enfermedad fue inesperada, pues en varios estudios se reporta una menor supervivencia libre de enfermedad para individuos con 
tumores con alta expresión de Bcl-2 $(12,13)$ mientras en otros no se reporta esta asociación (21-23).

La asociación de los niveles de expresión de Bax, Bcl-xL y Bad y la supervivencia ha sido poco evaluada. En varios estudios $(22,24,25)$ al igual que nosotros no encuentran asociación entre la expresión de Bax y la supervivencia, en contraste, Sohn y cols reportan asociación entre la expresión de Bax y supervivencia total y libre de enfermedad (21). En concordancia con nuestros resultados, Troutaud y cols encuentran que bajos niveles de expresión de Bad se asocian con la presencia de estados clínicos avanzados (16).

Se ha sugerido que la proteína Bad y los miembros similares que contienen sólo el dominio $\mathrm{BH}_{3}$ actúan corriente arriba de los miembros multidominio controlando de esta manera el destino celular. El dominio BH3 participa en la formación de heterodímeros con las proteínas anti-apoptóticas. Bcl2 y Bcl-xL se unen con gran afinidad al domino BH3 de la proteína Bad, a través del bolsillo hidrofóbico que se forma en sus superficies (26). Se ha propuesto que estas interacciones directas proteína-proteína neutralizarían las funciones anti-apoptóticas de Bcl-2 y sus homólogos como Bcl-xL (27). La proteína Bad parece ser importante en el desarrollo del LBDCG. En un modelo murino deficiente de Bad, los ratones con la edad desarrollan LBCGD de origen en centro germinal, esto sugiere un papel putativo de Bad en la patogénesis de estas neoplasias $(11,28,28)$. Se ha propuesto que Bad por sí sola no es suficiente para conducir a la muerte celular, aunque podría en su estado desfosforilado unirse a Bcl-2, disminuyendo el umbral de activación de otras proteínas pro-apoptóticas como Bak y Bax, y producir la muerte celular (28).

Es posible que la asociación entre altos niveles de expresión de Bad, Bcl-xL y Bcl-2 con la supervivencia libre de enfermedad se deba a la interacción que tienen entre sí durante la apoptosis. Los miembros anti- y pro-apoptóticos permanecen en distintos compartimientos subcelulares, pero ante una señal de muerte, las proteínas pro-apoptóticas pueden sufrir un cambio conformacional que les permite integrarse a las membranas donde residen las proteínas anti-apoptóticas $(29,30)$. La proteína Bad podría sufrir la desfosforilación y subsiguiente liberación del complejo con la proteína citosólica 14-3-3, resultando en la dimerización con Bcl-2 y Bcl-xL y la inactivación de las funciones de supervivencia de estas proteínas, resultando en la muerte celular (31). Aunque las tres proteínas se encuentren en niveles de expresión altos, el balance entre ellas puede resultar en la muerte celular y un aumento en la apoptosis en las células tumorales, reflejándose en una mayor supervivencia libre de recaídas. En este sentido, es interesante la observación de Abbott y cols quienes reportan que al tratar un línea celular derivada de un linfoma B no Hodgkin con una molécula orgánica pequeña se induce apoptosis con expresión incrementada de las proteínas Bcl-2, Bcl-xL y Bad (32).

Las asociaciones encontradas entre las proteínas de la familia Bcl-2 y el LBDCG han encaminado la investigación a la búsqueda de estrategias optimizadas de tratamiento que combinen el uso de agentes quimioterapéuticos con moléculas que neutralicen los efectos de las proteínas de la familia Bcl-2 y al desarrollo de ligandos para Bcl-2 y sus homólogos anti-apoptóticos. Una variedad de métodos incluyendo análisis computacional y tamización de productos naturales y sintéticos se han usado para identificar moléculas capaces de unirse a Bcl-2 o a Bcl-xL e inhibir sus funciones (27). En ese sentido y considerando que en un grupo de pacientes se observa una mejor supervivencia libre de enfermedad en presencia de Bad, a pesar de la expresión de altos niveles de $\mathrm{Bcl}-2$ y BcX-L, los pacientes con bajos niveles de expresión de Bad, podrían beneficiarse en un futuro con terapias orientadas a inhibir estas dos moléculas anti apoptóticas mediante la unión de moléculas especificas al dominio $\mathrm{BH} 3$.

\section{Agradecimientos}

Los autores de este estudio desean agradecer de manera especial a los grupos de Patología y de Hematología del Instituto Nacional de Cancerología.

\section{Financiación}

Este proyecto fue financiado por el Instituto Nacional de Cancerología. Bogotá, D.C., Colombia.

\section{Conflictos de intereses}

Los autores declaran que no existen conflictos de intereses con relación a este trabajo.

\section{Referencias}

1. Jaffe ES. The 2008 WHO classification of lymphomas: implications for clinical practice and translational research. Hematology. American Society of Hematology. Education Program. 2009; 523-531. 
2. Jaffe ES, Harris NL, Stein H, Campo E, Pileri S, Swerdlow SH. Introduction and overview of the Classification of the Lymphoid Neoplasias. In: Swerdlow SH, Campo E, Harris NL, Jaffe ES, Pileri SA, Stein H, Thiele J, Vardiman JW, eds. World Health Organization Classification of Tumours of Haematopoietic and Lymphoid Tissues. Lyon: IARC press, 2008: 158-166.

3. Wilder RB, Rodriguez MA, Medeiros LJ, Tucker SL, Ha CS, Romaguera JE, Pro B, Hess MA, Cabanillas F, Cox JD. International prognostic index-based outcomes for diffuse large B-cell lymphomas. Cancer 2002; 94, 3083-3088.

4. Lossos IS, Morgensztern D. Prognostic biomarkers in diffuse large B-cell lymphoma. Journal of Clinical Oncology. 2006; 24, 995-1007.

5. Rosenwald A, Staudt LM. Clinical translation of gene expression profiling in lymphomas and leukemias. Seminars in Oncology. 2002; 29, 258-263.

6. Alacacioglu I, Ozcan MA, Ozkal S, Piskin O, Turgut N, Demirkan F, Ozsan GH, Kargi A, Undar B. Prognostic significance of immunohistochemical classification of diffuse large B-cell lymphoma. Hematology. 2009; 14, 84-89.

7. Reed JC. Bcl-2 family proteins: regulators of apoptosis and chemoresistance in hematologic malignancies. Seminars in Hematology. 1997; 34, 9-19.

8. Cory S, Adams JM. The Bcl2 family: regulators of the cellular life-or-death switch. Nature Reviews. Cancer 2002; 2, 647-656.

9. Saez AI, Saez AJ, Artiga MJ, Perez-Rosado A, Camacho FI, Diez A, Garcia JF, Fraga M, Bosch R, RodriguezPinilla SM, Mollejo M, Romero C, Sanchez-Verde L, Pollan M, Piris MA. Building an outcome predictor model for diffuse large B-cell lymphoma. American Journal of Pathology. 2004; 164, 613-622.

10. Bairey O, Zimra Y, Shaklai M, Okon E, Rabizadeh E. $\mathrm{Bcl}-2, \mathrm{Bcl}-\mathrm{X}, \mathrm{Bax}$, and Bak expression in shortand long-lived patients with diffuse large B-cell lymphomas. Clinical Cancer Research. 1999; 5, 28602866.

11. Bai M, Skyrlas A, Agnantis NJ, Kamina S, Tsanou E, Grepi C, Galani V, Kanavaros P. Diffuse large Bcell lymphomas with germinal center B-cell-like differentiation immunophenotypic profile are associated with high apoptotic index, high expression of the proapoptotic proteins bax, bak and bid and low expression of the antiapoptotic protein bcl-xl. Modern Pathology. 2004; 17, 847-856.

12. Hill ME, MacLennan KA, Cunningham DC, Vaughan HB, Burke M, Clarke P, Di Stefano F, Anderson L, Vaughan HG, Mason D, Selby P, Linch DC. Prognostic significance of BCL-2 expression and bcl-2 major breakpoint region rearrangement in diffuse large cell non-Hodgkin's lymphoma: a British National Lymphoma Investigation Study. Blood 1996; 88, 1046-1051.

13. Kramer MH, Hermans J, Parker J, Krol AD, KluinNelemans JC, Haak HL, van GK, van Krieken JH, de JD, Kluin PM. Clinical significance of bcl2 and p53 protein expression in diffuse large B-cell lymphoma: a population-based study. Journal of Clinical Oncology. 1996; 14, 2131-2138.

14. Piris MA, Pezzella F, Martinez-Montero JC, Orradre JL, Villuendas R, Sanchez-Beato M, Cuena R, Cruz MA, Martinez B, Pezella F. p53 and bcl-2 expression in high-grade B-cell lymphomas: correlation with survival time. British Journal of Cancer 1994; 69, 337-341.

15. Wilson WH, Teruya-Feldstein J, Fest T, Harris C, Steinberg SM, Jaffe ES, Raffeld M. Relationship of p53, bcl-2, and tumor proliferation to clinical drug resistance in non-Hodgkin's lymphomas. Blood 1997; 89, 601-609.

16. Troutaud D, Petit B, Bellanger C, Marin B, GourinChaury MP, Petit D, Olivrie A, Feuillard J, Jauberteau MO, Bordessoule D. Prognostic Significance of BAD and AIF Apoptotic Pathways in Diffuse Large BCell Lymphoma. Clinical Lymphoma, Myeloma \& Leukemia. 2010; 10, 118-124.

17. Iqbal J, Neppalli VT, Wright G, Dave BJ, Horsman DE, Rosenwald A, Lynch J, Hans CP, Weisenburger DD, Greiner TC, Gascoyne RD, Campo E, Ott G, MullerHermelink HK, Delabie J, Jaffe ES, Grogan TM, Connors JM, Vose JM, Armitage JO, Staudt LM, Chan WC. BCL2 expression is a prognostic marker for the activated B-cell-like type of diffuse large B-cell lymphoma. Journal of Clinical Oncology. 2006; 24, 961-968.

18. Barrans SL, Carter I, Owen RG, Davies FE, Patmore RD, Haynes AP, Morgan GJ, Jack AS. Germinal center phenotype and bcl-2 expression combined with the International Prognostic Index improves patient risk stratification in diffuse large B-cell lymphoma. Blood 2002; 99, 1136-1143. 
19. Rantanen S, Monni O, Joensuu H, Franssila K, Knuutila $\mathrm{S}$. Causes and consequences of BCL2 overexpression in diffuse large B-cell lymphoma. Leukemia \& Lymphoma 2001; 42,1089-1098.

20. de JD, Xie W, Rosenwald A, Chhanabhai M, Gaulard P, Klapper W, Lee A, Sander B, Thorns C, Campo E, Molina T, Hagenbeek A, Horning S, Lister A, Raemaekers J, Salles G, Gascoyne RD, Weller E. Immunohistochemical prognostic markers in diffuse large B-cell lymphoma: validation of tissue microarray as a prerequisite for broad clinical applications (a study from the Lunenburg Lymphoma Biomarker Consortium). Journal of Clinical Pathology. 2009; 62, 128-138.

21. Sohn SK, Jung JT, Kim DH, Kim JG, Kwak EK, Park T, Shin DG, Sohn KR, Lee KB. Prognostic significance of bcl-2, bax, and p53 expression in diffuse large Bcell lymphoma. American Journal of Hematology. 2003; 73, 101-107.

22. Pagnano KB, Silva MD, Vassallo J, Aranha FJ, Saad ST. Apoptosis-regulating proteins and prognosis in diffuse large B cell non-Hodgkin's lymphomas. Acta Haematologica. 2002; 107, 29-34.

23. Ennishi D, Takeuchi K, Yokoyama M, Asai H, Mishima Y, Terui Y, Takahashi S, Komatsu H, Ikeda K, Yamaguchi M, Suzuki R, Tanimoto M, Hatake K. CD5 expression is potentially predictive of poor outcome among biomarkers in patients with diffuse large B-cell lymphoma receiving rituximab plus CHOP therapy. Annals of Oncology. 2008; 19, 1921-1926.

24. Gascoyne RD, Krajewska M, Krajewski S, Connors JM, Reed JC. Prognostic significance of Bax protein expression in diffuse aggressive non-Hodgkin's lymphoma. Blood 1997; 90, 3173-3178.

25. Hasselblom S, Hansson U, Olsson M, Toren L, Bergstrom A, Nilsson-Ehle H, Andersson PO. High immunohistochemical expression of p-AKT predicts inferior survival in patients with diffuse large B-cell lymphoma treated with immunochemotherapy. British Journal of Haematology 2010; 149, 560-568.

26. Yang E, Zha J, Jockel J, Boise LH, Thompson CB, Korsmeyer SJ. Bad, a heterodimeric partner for Bcl$\mathrm{XL}$ and Bcl-2, displaces Bax and promotes cell death. Cell 1995; 80, 285-291.

27. Rutledge SE, Chin JW, Schepartz A. A view to a kill: ligands for Bcl-2 family proteins. Current Opinion in Chemical Biology 2002; 6, 479-485.

28. Ranger AM, Zha J, Harada H, Datta SR, Danial NN, Gilmore AP, Kutok JL, Le Beau MM, Greenberg ME, Korsmeyer SJ. Bad-deficient mice develop diffuse large B cell lymphoma. Proceedings of the National Academy of Sciences.U.S.A 2003; 100, 9324-9329.

29. Degli EM, Dive C. Mitochondrial membrane permeabilisation by Bax/Bak. Biochemical and Biophysical Research Communications.. 2003; 304, 455-461.

31. Zhou H, Hou Q, Chai Y, Hsu YT. Distinct domains of Bcl-XL are involved in Bax and Bad antagonism and in apoptosis inhibition. Experimental Cell Research. 2005; 309, 316-328.

32. Abbott DR, Abbott RT, Jenson SD, Fillmore GC, ElenitobaJohnson KS, Lim MS. Apoptosis of $\mathrm{t}(14 ; 18)$-positive lymphoma cells by a Bcl-2 interacting small molecule. Journal of Hematopathology. 2009; 2, 113-119. 